\title{
Mild anemia as a protective factor against pregnancy loss
}

\author{
L.O. Buzyan* \\ Clinic of the South Ural State Medical University, Maternal and Child Health Care Department, \\ Chelyabinsk, Russia
}

*Corresponding author. E-mail: liduhe@inbox.ru

BACKGROUND: Iron deficiency anemia is traditionally considered to be a pathological condition during pregnancy. According to the standards, prescription of iron supplements to pregnant women is required at hemoglobin levels of $110 \mathrm{~g} / \mathrm{l}$ and lower. Numerous studies at different periods showed the relationship of anemia and premature birth [5], preeclampsia [1], low birth-weight [2]. Meanwhile, physiological hemodilution carries in pregnancy an important adaptive function. It is well known, that in the second half of pregnancy physiological hypercoagulability develops (increased activity of the plasma clotting factors, platelet aggregation, decreased activity and blood concentrations of physiological anticoagulants) aimed at implementing adequate hemostasis in labor. Under these conditions, moderate hemodilution is an effective mechanism for preventing the development of severe disseminated intravascular coagulation (DIC) in labor, during surgery, in various forms of obstetric pathology.

OBJECTIVE: To study the effect of anemia of varying severity on the pregnancy course and outcomes.

METHODS: We conducted a "case-control" study based on cohort. The study included 421 pregnant women who received outpatient care under the monitoring of pregnancy in the antenatal clinic of the South Ural State Medical University and the antenatal clinic of Chelyabinsk Clinical Hospital №6. The inclusion period was from January to March 2014. Inclusion criteria were: confirmed pregnancy, informed consent to participate in the study. Exclusion criteria were multiple pregnancy, induced pregnancy, late first appearance in the antenatal clinic (after 25 weeks' gestation), change of residence and medical supervision during pregnancy, the presence of severe mental disorders, severe somatic diseases in decompensation stage, HIV infection, cancer, active tuberculosis. Study design complies with the legislation of the Russian Federation, international ethical standards and was approved by the Ethics Committee of the South Ural State Medical University. Prospective observation was conducted. All pregnancy complications were registered, in particular, preeclampsia, placental insufficiency (violation of utero-placental blood flow), the presence and severity of anemia during pregnancy, as well as outcomes: the duration and mode of delivery, fetal weight at birth. The criteria for the diagnosis of anemia during pregnancy were: decrease of hemoglobin levels below $110 \mathrm{~g} / 1$. All pregnant women with confirmed anemia were treated with iron supplements. After the completion of follow-up the odds ratios analysis of presenting anemia was performed in subgroups: 1) a patient gave birth to a live child, and patient with the loss of the fetus at various stages of gestation; 2) patients who have given birth in time, and patients with premature birth; 3) patients with placental insufficiency (violation of utero-placental blood flow) during pregnancy and patients without placental insufficiency; 4) patients with preeclampsia and patients without pre-eclampsia; 5) patients who have delivered live births weighing less than 2,500 grams and birth weight over 2500 . In each case, odds ratios (ORs) and confidence intervals $(95 \% \mathrm{CI})$ were calculated. Statistical calculations were performed using the software package SPSS 22.0. 
RESULTS: Among all patients $(n=421)$ anemia of varying severity has been diagnosed in $190(45.13 \%)$, including mild anemia in 161 (38.24\%), moderate or severe anemia - in 29 patients $(6.88 \%)$. In 16 (3.8\%) cases, the pregnancy resulted in miscarriage or fetal death. Premature birth of live fetus before 34 weeks of gestation was recorded in 13 cases $(3.2 \% ; n=405)$. Preeclampsia was diagnosed in 15 women (3.56\%). Live births with weights less than $2,500 \mathrm{~g}$ were registered in 23 cases $(5.67 \% ; n=405)$.

An analysis of odds ratios was performed to compare the groups of women with anemia of any severity and without anemia, with mild anemia compared with women without anemia, and moderate/ severe anemia compared to women with mild anemia or without anemia.

According to our results, there was a statistically significant reduction in the chance of having anemia of any severity in patients whose pregnancy was completed by fetal loss. For mild anemia odds ratio in these subgroups was even lower. Thus, the odds of having mild anemia in the group of women who completed a pregnancy to a live birth, was $90.3 \%$, which may indicate a protective role of mild anemia against the loss of the fetus.

In all other cases, statistically significant results were not received. Thus, we didn't receive significant positive association between anemia and development of preeclampsia, placental insufficiency during pregnancy, low birth weight and premature birth. There are other publications that show a protective role of anemia in pregnancy. Case control study [3] showed a protective role of anemia against the development of pre-eclampsia $(n=636, p=0,01)$. In [4] anemia was a protective factor against stillbirth. There is evidence [6] of the higher risk of stillbirth in women with high hemoglobin level (146 g/l and above), while the link of the risk of stillbirth with anemia has not been confirmed $(n=1404)$.

CONCLUSIONS: Our data suggest a protective role of mild anemia during pregnancy in relation to pregnancy loss.

Keywords: Anemia, pregnancy, pregnancy loss, mild anemia, protective factor

Conflict of interest statement: None.

\section{References}

[1] Endeshaw M, Abebe F, Bedimo M. Diet and Pre-eclampsia: A Prospective Multicentre Case-Control Study in Ethiopia. Midwifery. 2015;31(6):617-24. doi: 10.1016/j.midw.2015.03.003. Epub 2015 Mar 10.

[2] Haider BA, Olofin I, Wang M, Spieqelman D, Ezzati M, Fawzi WW. Anemia, prenatal iron use, and risk of adverse pregnancy outcomes: Systematic review and meta-analysis. BMJ. 2013;346:f3443. doi: 10.1136/bmj.f3443

[3] Kashanian M, Baradaran HR, Bahasadri S, Alimohammadi R. Risk factors for pre-eclampsia: A study in Tehran, Iran. Arch Iran Med. 2011;14(6). P. 412-5.

[4] Stephansson O, Dickman PW, Johansson A, Cnattinquis S. Maternal hemoglobin concentration during pregnancy and risk of stillbirth. JAMA. 2000;284(20). P. 2611-7.

[5] van den Broek NR, Jean-Baptiste R, Neilson JP. Factors associated with preterm, early preterm and late preterm birth in Malawi. PLoS One. 2014;9(3):e90128. doi: 10.1371/journal.pone.0090128. eCollection 2014

[6] Zhang Q, Ananth CV, Rhoads GG, Li Z. The impact of maternal anemia on perinatal mortality: A population-based, prospective cohort study in China. Ann Epidemiol. 2009;19(11):793-9. doi: 10.1016/j.annepidem.2009.06.002. Epub 2009 Aug 3. 